\title{
SÍNDROME DA FRAGILIDADE NO IDOSO (SFI) COMO FATOR DE PIOR PROGNÓSTICO DA COVID-19
}

Kimberly Mayara Gouveia Bezerra; Universidade Federal de Pernambuco - UFPE; kimberly.gouveia@ufpe.br

Crislayne Maria Berto; Universidade Federal de Pernambuco - UFPE; crislayne.berto@ufpe.br Fernando Arthur Alves da Silva; Universidade Federal de Pernambuco - UFPE; fernando.arthur@ufpe.br Leiliane Moraes dos Santos Silva; Universidade Federal de Pernambuco - UFPE; leiliane.moraes@ufpe.br Ana Paula de Oliveira Marques; Universidade Federal de Pernambuco - UFPE; ana.marques@ufpe.br

\section{RESUMO}

Introdução: $\mathrm{O}$ envelhecimento tem sido considerado um fator de risco para a infecção pelo novo coronavírus, apresentando desfechos e prognósticos clínicos negativos, em comparação com indivíduos mais jovens. Dentre os fatores envolvidos nessa problemática, destaca-se a Síndrome da Fragilidade no Idoso (SFI), uma condição de vulnerabilidade fisiológica causada pela diminuição da reserva homeostática e pelo declínio da resistência e a energia do organismo. Objetivo: Analisar na literatura as evidências sobre a SFI como fator de pior prognóstico da COVID-19. Métodos: Realizou-se uma revisão integrativa nas bases de dados Literatura Internacional em Ciências da Saúde (MEDLINE), Scientific Electronic Library Online (SciELO), Centro Latino-Americano e do Caribe de Informação em Ciências da Saúde (LILACS) e Pubmed utilizando a combinação dos descritores "COVID-19", "frailty syndrome" e "elderly". Do total de 70 artigos encontrados, 19 foram pré-selecionados e 8 artigos se enquadraram nos critérios de elegibilidade. Resultados: Evidenciou-se que a idade cronológica e a presença de multimorbidade não parecem ser os fatores mais críticos de risco para resultados desfavoráveis da COVID-19 em idosos. O funcionamento é um elemento-chave na determinação do prognóstico desses pacientes. Além disso, a avaliação da fragilidade pré-mórbida no idoso, quando devidamente analisada, é uma medida essencial que pode orientar o estabelecimento de metas de cuidado. Conclusão: A SFI, por causar declínio funcional, diminui a capacidade do organismo de enfrentar desfechos negativos à saúde, como quedas, perda funcional, hospitalização, com maior probabilidade de morte. Por isso, a identificação de idosos frágeis torna-se fundamental no cuidado à pessoa idosa com COVID-19.

Palavras-chave: Idoso; Síndrome da fragilidade; COVID-19; Prognóstico. 